\title{
La pandémie, source de risques et d'opportunités pour la mise en place de pratiques de transition collaboratives lors de l'entrée à l'école
}

\section{Stéphanie Duval}

Professeure agrégée

Université Laval

Joanne Lehrer

Professeure régulière

Université du Québec en Outaouais

Florence Pirard

Professeure

Université de Liège

Marie Housen

Doctorante

Université de Liège stephanie.duval@fse.ulaval.ca

joanne.lehrer@uqo.ca

florence.pirard@uliege.be

marie.housen@uliege.be

\section{Résumé}

La transition vers l'école est une étape importante dans la vie d'un enfant et de sa famille, laquelle amène un lot d'émotions, d'attentes et d'inquiétudes, voire d'ajustements de la part de tous les acteurs y étant impliqués (enfants, parents, personnel éducateur et enseignant, etc.). Des recherches réalisées dans plusieurs contextes internationaux ont montré l'importance de mettre en place des initiatives permettant à l'enfant et à sa famille de vivre des transitions harmonieuses, notamment celles qui se déroulent à l'entrée de la période préscolaire, puisque cette dernière conditionne son bien-être et influence les autres moments de passage vécus tout au long de son parcours scolaire. En contexte de pandémie, en des temps où la distanciation sociale et physique est préconisée, comment les pratiques de transition sont-elles différentes de celles mises en place auparavant? Ce contexte de crise peut-il générer des transformations de pratiques collaboratives qui assurent une continuité dans les expériences vécues par l'enfant? Cet article de synthèse traite de ces questions, en considérant le contexte de pandémie actuel comme source de possibles risques mais aussi d'occasions pour la mise en place de pratiques de collaboration entre l'école et la famille.

Mots clés : Transition ; École maternelle ; Collaboration ; Pandémie 


\section{Introduction}

Les meilleures manières de soutenir l'enfant en début de scolarité font l'objet de débats dans de nombreux pays et régions où un intérêt grandissant est porté aux premières transitions scolaires. Pour cause, les premières transitions vers l'école (ayant lieu entre 2,5 - 6 ans selon le système éducatif des pays) sont primordiales à considérer (Conseil supérieur de l'éducation [CSÉ], 2012). Elles marquent, pour la plupart des enfants et leur famille, le début d'une histoire scolaire et relationnelle qui s'établit entre eux et l'école (Duval et Lehrer, sous presse; Groupe de travail régional en transition scolaire, 2018). Elles ne consistent pas seulement en l'ajustement des différents acteurs (p. ex. enfants, parents) au milieu éducatif, elles impliquent aussi la déconstruction de repères quant aux expériences passées (Ackesjö, 2014) et l'importance d'une reconnaissance réciproque de la culture familiale et scolaire (Dockett, Griebel, \& Perry, 2017).

Il est reconnu que la façon dont l'enfant vit ses premiers moments lors de l'entrée à l'école influence sa capacité d'ajustement lors des transitions futures (Fabian \& Dunlop, 2007 ; Ministère de l'Éducation, du Loisir et du Sport [MELS], 2010 ; Petrakos \& Lehrer, 2011; Ruel, Moreau, Bérubé, \& April, 2015) et affecte son engagement initial à l'école ainsi que ses apprentissages ultérieurs (Fabian, 2002 ; Ruel, Bérubé, Moreau, \& April, 2018 ; White \& Sharp, 2007). Des recherches démontrent également que l'adaptation de l'enfant est plus facile si ses parents se sentent prêts, soutenus par la variété d'activités offertes par l'école pour les accueillir (Bérubé, Ruel, April, \& Moreau, 2018; Giallo, Kienhuis, Treyvaud, \& Matthews, 2008).

Dans ce contexte particulier de pandémie, les différents acteurs impliqués dans la transition vers l'école (p. ex., enfants, parents, enseignants, éducateurs en service de garde extrascolaire) ont dû déployer des efforts supplémentaires pour s'ajuster à cette situation et aux mesures sanitaires exceptionnelles recommandées. Selon l'Organisation des Nations unies pour l'éducation, la science et la culture [UNESCO] (2020), la pandémie de COVID-19 a entraîné des changements importants dans le monde de l'éducation. Près de $90 \%$ de la population étudiante mondiale, soit plus de 1,5 milliard d'apprenants dans 165 pays, ont vu leurs expériences d'apprentissage et de développement perturbées par des précautions et des politiques mises en œuvre pour endiguer la propagation de la maladie. Plus spécifiquement à l'égard de l'éducation à la petite enfance, cette crise sanitaire internationale a précipité des changements sans précédent dans la vie des enfants et leurs familles, de même que dans celle du personnel éducateur et enseignant œuvrant au service des jeunes enfants (National Association for the Education of Young Children [NAEYC], 2020 ; Zero to Three, 2020).

Les enfants sont particulièrement vulnérables aux effets des catastrophes communautaires et des autres crises et événements traumatisants. Cela est dû à leur manque d'expériences, de compétences et de ressources qui leur permettraient de répondre de manière indépendante à leurs besoins en matière de santé développementale, socioémotionnelle, mentale et comportementale (Schonfeld, Demaria, \& Kumar, 2020). Ainsi, les crises telles que la COVID-19 peuvent avoir des effets à court et à long terme sur le fonctionnement psychologique, l'adaptation émotionnelle, la santé et les trajectoires de développement des enfants, et peuvent même avoir des répercussions sur leur santé et leur fonctionnement social et psychologique à l'âge adulte (Schonfeld et al., 2020). Par ailleurs, notons que les enfants démontrent également une résilience face aux évènements critiques (Lemay, 1998), une capacité à influer sur leur environnement, surtout lorsque des conditions sont mises en place pour les soutenir. On peut voir ici l'intérêt d'offrir une vision alternative au discours inscrit dans une logique uniquement déficitaire de l'enfant, sans minimiser les défis que ces derniers, de même que leurs familles et les adultes qui les entourent (p. ex.

@2021 Duval, Lehrer, Pirard, Housen

DOI https://doi.org/10.51657/ric.v5i1.41066 
enseignants) ont à relever dans ce contexte de pandémie. On soutient, dans cette vision, que l'enfant est un être compétent, riche en potentiel et un participant actif dans le processus de la transition vers l'école (Dahlberg, Moss, \& Pence 2013; Disanto \& Berman, 2012; Einarsdottir, Dockett, \& Perry, 2009).

En somme, des recherches réalisées à l'international (p. ex. Hopps, 2014 ; Smith \& Maher, 2016) insistent sur l'importance de mettre en place des initiatives permettant à l'enfant et sa famille de vivre des transitions harmonieuses, notamment celles qui se déroulent pendant la période préscolaire. En ces temps où la distanciation sociale et physique est mise de l'avant, il convient de mentionner que la crise de la COVID-19 ajoute un aspect de nouveauté à la transition qui s'avère déjà singulière pour les enfants, parents, éducateurs et enseignants. Comment les pratiques de transition et de collaboration peuvent-elles être déployées entre les différents acteurs, afin d'assurer une continuité dans les expériences vécues par l'enfant? C'est ce dont il est question dans cet article qui à partir d'une revue sélective d'études consacrées à la notion polysémique de transition et aux pratiques qui s'y rapportent dans l'accueil et l'éducation des jeunes enfants, propose d'abord une grille d'analyse qui en éclaire les significations en temps de non pandémie pour ensuite les mettre en perspective dans le contexte de crise de la COVID-19. In fine, cet article propose un cadre de réflexion utile à la recherche et à la pratique (Lehrer, Bigras. Charron, et Laurin, à paraitre), qui relève dans une démarche dialectique les occasions et les risques pour la mise en œuvre de pratiques de transition et de collaboration dans différents contextes au Québec et en Europe. L'appui d'articles de presse et d'entrevues de chercheurs qui sont intervenus dans les débats a également été utilisé, notamment en raison du fait qu'il y a encore, à l'heure, peu d'études publiées sur le sujet.

\section{La transition : une question centrale}

La notion de transition : de quoi s'agit-il?

De manière générale, une transition représente une période de changement chez une personne, impliquant des transformations qualitatives plus ou moins importantes dans sa vision du monde, son concept de soi, ses relations et ses rôles (Cowan, 1991 ; Schlossberg, 2005). Tout être humain est amené, au cours de sa vie, à rencontrer des transitions plus ou moins marquantes telles qu'un déménagement, la perte d'un proche, une intégration professionnelle, etc. La transition ne correspond pas à l'évènement déclencheur, mais bien à ce processus de passage d'un état à l'autre. Il s'agit ainsi du stade intermédiaire se déroulant entre deux situations.

Selon Schlossberg (2005), les transitions peuvent être classées selon leur degré de prévisibilité. Trois types de transition sont alors identifiés. Tout d'abord, on retrouve les transitions anticipées qui correspondent à des évènements importants et prévisibles auxquels l'être humain devra faire face et pour lesquels il aura pu se préparer (p. ex. un mariage, la retraite, le fait de devenir parent, etc.). L'entrée à l'école maternelle pourrait être qualifiée de transition anticipée étant donné que celle-ci est planifiable et peut donc être préparée. Ensuite, il y a les transitions non anticipées qui réfèrent aux évènements soudains, brusques et non attendus, qui sont caractérisés par peu de préparation pour y faire face. On peut penser à une catastrophe naturelle, à un accident, à un décès, etc. Si l'entrée à l'école maternelle n'est pas préparée, elle pourrait être vécue par les enfants comme étant une transition non anticipée. Enfin, les transitions «non-évènements" 
renvoient au dernier type répertorié par Schlossberg (2005), lesquelles renvoient aux évènements attendus qui n'ont finalement pas lieu (p. ex. ne pas se marier, ne pas être promu, être incapable de fonder une famille, ne pas trouver l'emploi espéré, etc.).

De plus, les transitions peuvent être regroupées selon qu'elles sont horizontales ou verticales. Les transitions horizontales correspondent aux différents changements qui peuvent survenir au cours d'une même journée, tel qu'un changement de local, d'activités, etc. Elles nécessitent une attention particulière, mais elles ont un caractère moins marquant que les transitions verticales qui sont moins fréquentes et dont les changements associés ont un caractère durable comme l'entrée dans le système scolaire (Ruel, 2009). La transition vers la maternelle représente une importante rencontre entre différents milieux de socialisation de l'enfant (p. ex. la famille, le milieu de garde, l'école) (Bérubé et al., 2018; Cotnoir, 2015), est qualifiée de verticale, car elle concerne un passage entre différentes étapes de vie, comme entre la famille et l'école, ou le milieu de garde et la maternelle. Les changements vécus pendant cette période de passage ont un caractère durable, puisqu'ils modifient qualitativement une composante de la vie d'une personne, ici l'enfant et sa famille (Duval et Lehrer, sous presse).

En somme, les périodes de transition constituent des moments sensibles pour le développement de la personne. Elles peuvent à la fois entraîner une période de vulnérabilité accrue, ainsi que des potentialités et des occasions qui rendent possible l'émergence de nouvelles habiletés cognitives, sociales, personnelles, etc. De plus, ces potentialités et opportunités permettent à l'enfant d'accroitre son sentiment de compétence, son sentiment d'appartenance, son niveau de bien-être à l'école, la construction de nouvelles amitiés, l'établissement d'une relation positive avec l'enseignante, etc. Lorsque la transition est planifiée et soutenue de manière adéquate, notamment en misant sur la continuité, elle offre des ressources d'ajustements qui peuvent aider la personne à affronter de nouvelles périodes de changement tout au long de sa vie, lors d'expériences ultérieures de transition. Les transitions, bien que se déroulant sur une période de temps déterminée, vont donc s'inscrire à long terme dans le parcours de vie de la personne (Dockett \& Perry, 2015).

\section{La notion de continuité}

Un peu partout à travers le monde, des chercheurs (p. ex. Ballam, Perry, \& Garpelin, 2017 ; Organisation de coopération et de développement économiques [OCDE], 2017) abordent l'importance de la continuité dans le processus de transition, notamment pendant les expériences vécues à la petite enfance. Le fait de concevoir la transition vers l'école comme une continuité dans les expériences et les apprentissages des enfants permet de tirer tous les bénéfices de la nouveauté qu'elle comporte (Curchod-Ruedl \& Chessex-Viguet, 2012). Pour ce faire, il importe d'agir sur plusieurs composantes relatives à chaque enfant, à sa famille et à l'école (Rimm-Kaufmann \& Pianta, 2000). Cette continuité implique notamment la mise en place et le maintien de relations entre les différents environnements fréquentés par l'enfant et sa famille.

Dans cet ordre d'idées, le Tableau 1 présente les types de continuité définis par Dockett et Perry (2007). 
Revue internationale du CRIRES : innover dans la tradition de Vygotsky (2021), vol 5, no 1

\section{Tableau 1.}

Les types de continuités d'expérience proposés par Dockett et Perry (2007)

\begin{tabular}{|c|c|}
\hline Continuité des expériences & Définition \\
\hline Continuité philosophique & $\begin{array}{l}\text { Vise le développement d'une compréhension commune sur les approches des } \\
\text { praticiens. Le regard est porté sur l'enfant, les manières dont il apprend et se } \\
\text { développe, afin d'arrimer les attentes du personnel éducateur et enseignant au } \\
\text { regard des enfants. }\end{array}$ \\
\hline $\begin{array}{l}\text { Continuité curriculaire } \\
\text { (ou des programmes) }\end{array}$ & $\begin{array}{l}\text { Concerne l'établissement de liens entre les contenus traités, les situations } \\
\text { d'apprentissage et de développement ainsi que les compétences observées dans } \\
\text { les milieux éducatifs. Ce type de continuité permet de reconnaitre la valeur des } \\
\text { différents programmes (p. ex. milieu de garde, maternelle), de même que la } \\
\text { contribution unique des contextes et des personnes impliquées dans la } \\
\text { transition. }\end{array}$ \\
\hline Continuité pédagogique & $\begin{array}{l}\text { Réfère à la cohérence entre les pratiques éducatives utilisées afin de soutenir les } \\
\text { apprentissages des enfants. On peut voir des liens concrets entre la continuité } \\
\text { des programmes et la continuité pédagogique. Selon l'OCDE (2018), ces deux } \\
\text { types de continuité permettent d'éviter une variation importante dans les } \\
\text { pratiques éducatives déployées, afin de favoriser une approche commune entre } \\
\text { les contextes éducatifs. }\end{array}$ \\
\hline Continuité physique & $\begin{array}{l}\text { Vise à atténuer les différences liées à l'aménagement spatial : la taille des } \\
\text { bâtiments, l'étendue du terrain de jeu, la disponibilité des outils ressources } \\
\text { éducatives, le matériel de manipulation, l'aménagement des aires de jeu, etc. }\end{array}$ \\
\hline Continuité organisationnelle & $\begin{array}{l}\text { Permet d'assurer une cohérence quant à l'horaire de la journée, le ratio } \\
\text { adulte/enfants, les normes réglementaires et les conditions d'emploi du } \\
\text { personnel éducateur et enseignant. }\end{array}$ \\
\hline Continuité administrative & $\begin{array}{l}\text { Permet d'encourager la centralisation des services au sein d'une même } \\
\text { organisation, afin de régir les aspects administratifs, de même qu'à appliquer } \\
\text { les mêmes politiques au sein des contextes éducatifs. }\end{array}$ \\
\hline
\end{tabular}

À ces types de continuité s'ajoute celle reliée à l'enfant, soit une continuité de développement. Celle-ci ne porte pas sur les expériences vécues, mais sur l'idée de favoriser l'apprentissage et le développement de tous les enfants, en valorisant d'abord leurs acquis en termes de connaissances, de compétences et d'attitudes, et en reconnaissant leurs forces et leurs besoins. Au-delà des expériences vécues dans le milieu éducatif, la continuité de développement permet de centrer son regard sur l'enfant et son unicité, soit les caractéristiques qui lui sont propres et qui lui permettent de s'ajuster au passage vers un nouvel environnement (Dockett \& Perry, 2017 ; Moll, Amanti, Neff, \& Gonzalez 1992). Selon l'OCDE (2018), la continuité de développement englobe aussi les pratiques de transition et de collaboration qui visent à impliquer les familles et les enfants dans le processus de passage à l'école. 
Duval, Lehrer, Pirard, Housen

\section{Pratiques de transition collaboratives}

Afin de favoriser la continuité entre les différents milieux de vie de l'enfant (p. ex. la famille et l'école) (Broström, 2002 ; Rimm-Kaufmann \& Pianta, 2000), des pratiques de transition peuvent être mises en place avant, pendant et après la rentrée scolaire, de manière à soutenir l'enfant et sa famille dans le passage vers l'école. Les pratiques de transition sont définies comme des activités planifiées et organisées par différents acteurs (p. ex. enseignants de maternelle, personnel de l'accueil extrascolaire, direction scolaire, etc.) dont l'un des objectifs est de développer des relations de confiance réciproque avec les familles (Athola et al., 2016; Cantin, 2005; Deslandes \& Jacques, 2004). Dans la perspective finlandaise, pays où le système éducatif est intégré, l'expression pratique de transition a le sens de collaboration en période de transition, ce qui met en évidence la recherche d'une réciprocité éducative dans le processus de transition entre les contextes. Elles permettent ainsi de tisser des liens entre les expériences antérieures vécues par l'enfant et les expériences nouvelles qu'il vivra lors de la transition vers la maternelle.

Caractérisé par une offre divisée de services, le Québec fait partie des pays pionniers dans la mise en place de telles pratiques (Cotnoir, 2005; Petrakos \& Lehrer, 2011; Bérubé et al., 2018) comparativement à d'autres contextes comme la France (Garnier \& Brougère, 2017), la Belgique (Housen \& Royen, 2019) ou la Suisse (Conus, 2015). Les visites entre les acteurs des milieux éducatifs impliqués dans la transition (p. ex. milieux de garde - Centre de la petite enfance au Québec et classe de maternelle 5 ans) représentent une des pratiques de transition et de collaboration implémentée. On peut aussi penser à des visites de la classe que va fréquenter l'enfant, des réunions d'information avec les parents, la participation des enfants et de leur parent dans des activités organisées par l'école, comme un pique-nique, une collaboration entre les professionnelles et les parents, etc. (Ministère de l'Éducation du Québec, 2003 ; MELS, 2010 ; OCDE, 2018). De plus, des rencontres ou les partages d'informations entre le milieu de garde et l'école représentent également des pratiques observées dans les milieux, de même que l'établissement de relations entre le personnel éducateur et enseignant (p. ex. personnel du service de garde en milieu scolaire et enseignants de maternelle).

La mise en place de telles pratiques de transition a largement été étudiée sur le plan international. Ainsi au Québec, un intérêt a été observé quant aux premières transitions scolaires et aux pratiques qui les facilitent. Des initiatives ont d'ailleurs vu le jour, tel que la création du Guide pour soutenir une première transition scolaire de qualité (MELS, 2010), qui a fait également l'objet d'une étude d'implantation (Ruel et al., 2015). Ces travaux reconnaissent la nécessité de considérer l'expérience des jeunes enfants avant, pendant et après toute période de transition, peu importe la culture dans laquelle ils grandissent. En fait, ces études montrent les nombreuses façons dont les systèmes actuels façonnent les expériences des enfants (Deslandes, 2013 ; Jacques \& Deslandes, 2002). En Europe, un intérêt y a aussi été porté à travers les années (Dunlop, 2018 ; Rayna \& Garnier, 2017); des études ont notamment été menées sur le rôle de l'enseignant (McCail, 1996), la notion de continuité (Dunlop, 1998), la prise en compte des perspectives des enfants et des parents (Hughes et al., 2015 ; Petrakos \& Lehrer, 2011), etc.

De plus, des travaux ont montré que les pratiques de transition doivent être réfléchies et planifiées de manière intentionnelle afin d'influencer la capacité d'ajustement de l'enfant et de sa famille. Pour cause, la collaboration entre les parents et le personnel enseignant est associée à une meilleure communication et à une plus grande confiance, facteurs qui soutiennent à leur tour la

@2021 Duval, Lehrer, Pirard, Housen

DOI https://doi.org/10.51657/ric.v5i1.41066

CR*̋̈RES 
transition des enfants vers l'école (Dockett \& Perry, 2001 ; Jacques \& Deslandes, 2002 ; KraftSayre \& Pianta, 2000). En outre, le fait d'encourager la participation des parents lors de la transition vers l'école peut les aider à réduire leur stress (Malsch, Green, \& Kothari, 2011). La construction de relations de confiance réciproque entre les enseignants et les familles se développe au travers des occasions d'échange fréquentes et de qualité (Deslandes, Bastien, \& Lemieux, 2004 ; Deslandes \& Jacques, 2004 ; Jacques \& Deslandes, 2002).

Ainsi, il importe de s'interroger sur la manière dont ces pratiques de transition collaboratives sont réalisées. En ce sens, Conus (2017) a montré qu'elles peuvent devenir normalisantes, c'est-à-dire qu'elles tendent à ne pas être individualisées, ni ajustées aux besoins des enfants et leur famille. Pourtant, des études ont montré l'importance de mettre en place des pratiques de transition individualisées et de les intensifier; une rencontre individuelle avec chaque enfant et ses parents en début d'année aura plus d'effet qu'une rencontre avec tous les parents en grand groupe (Petrakos \& Lehrer, 2011, 2011 ; Wildenger \& McIntyre, 2011), tout comme un appel téléphonique aura plus d'effet que la même lettre envoyée à chaque enfant pendant l'été. De plus, les démarches d'information unidirectionnelle (p. ex. les enseignantes qui envoient de l'information aux parents) ne favorisent pas le dialogue entre les différents acteurs pourtant nécessaire à une continuité de développement. Il importe ainsi de favoriser la mise en place de pratiques de collaboration bidirectionnelle, qui permettent de soutenir le dialogue entre les intervenants gravitant autour de l'enfant, pour favoriser une continuité de développement.

Ce souci de travailler en collaboration et en concertation est cohérent avec la perspective écologique et dynamique [PÉD] de Rimm-Kaufmann et Pianta (2000) souvent abordé dans les travaux sur la transition scolaire (p. ex., Jacques \& Deslandes, 2002). La PÉD permet de prendre en compte les différents milieux de vie dans lesquels l'enfant évolue, ainsi que les relations qui y existent. En privilégiant la PÉD, ce sont les acteurs qui soutiennent l'enfant qui l'accueillent dans le nouveau milieu avec ses particularités, qui l'accompagnent selon son niveau développemental, à son rythme, en cohérence avec le milieu de référence (la famille, le milieu de garde). Il s'agit ainsi de veiller à ce que l'école soit prête à accueillir tous les enfants (Broström, 2002) dans une visée d'équité et de lutte contre les inégalités sociales dans l'éducation (Housen \& Royen, 2019). Ces actions, qui s'opèrent dès l'entrée à la maternelle, semblent particulièrement importantes dans le contexte actuel de la pandémie, car la crise sanitaire mondiale semble exacerber certaines iniquités et inégalités notamment par l'attente d'une scolarisation dans l'espace familial (Plateforme d'échange, de recherche et d'intervention sur la scolarité : persévérance et réussite [PÉRISCOPE], 2020 ; Wagnon, 2020).

Puisque la première rentrée scolaire amène déjà un lot d'émotions, d'attentes et d'inquiétudes, voire d'ajustements de la part des acteurs impliqués (Dockett \& Einarsdottir, 2017 ; Van Laere \& Vandenbroeck, 2017), il semble primordial de se questionner sur les risques et les occasions qu'apporte celle effectuée dans un contexte de pandémie, car les praticiens et les chercheurs pensent que les perturbations de la planification de la transition pourraient avoir un effet sur la mise en place de liens entre la maison et l'école, sur la confiance des enfants dans leurs capacités et sur l'engagement parental (Chantier montréalais Transition vers l'école \& Ruel, 2020). Pour cause, lorsque des drames sociaux frappent le monde, des répercussions peuvent être observées chez les enfants, malgré la volonté des adultes de les protéger (Marinova, Dumais, \& Drainville, 2020). Il est possible que l'anxiété ressentie par les parents dans de telles circonstances 
influence la capacité d'ajustement des enfants à certaines situations, comme l'entrée à l'école, en raison des mesures de confinement. Si pour certains, ce moment de transition est vécu positivement, d'autres ressentent de l'instabilité et de l'insécurité.

\section{La pandémie, une crise qui bouleverse les pratiques}

Si le terme crise est actuellement utilisé pour désigner une période de difficulté, étymologiquement, il trouve son origine dans le verbe grec krinein signifiant « séparer », " juger », « choisir », « décider », mais aussi « se battre », " se mesurer aux autres » (Dechêne \& Dupont, 2019). Ce terme polysémique a été repris au cours des siècles par différentes disciplines dont la médecine, le droit, l'histoire, et la sociologie. Au sens sociologique, la crise s'entend comme une «[...] situation anomique de perturbations affectant finalement le système social dans son ensemble » (Ferry, 2014, p.52). Selon cette définition, la pandémie due à la COVID-19 est une crise qui a bouleversé l'ensemble du système social, dont le secteur de l'éducation comme le montrent les analyses récentes menées au Québec et en Europe, notamment en Fédération Wallonie-Bruxelles $(\mathrm{FW}-\mathrm{B})^{1}$ où trois études successives financées par le gouvernement étudient depuis 2018 l'émergence des pratiques de transition lors de l'entrée à la maternelle (Housen \& Royen, 2019 ; Housen, Royen \& Pirard, 2020).

\section{Des bouleversements à la maison...}

Au printemps 2020, sur le plan international, les parents et les enseignants ont dû s'adapter rapidement aux recommandations émises par l'Organisation mondiale de la santé [OMS] et la Direction de la Santé publique de leur pays, sans pouvoir anticiper les impacts potentiels de ces recommandations. Plusieurs parents ont temporairement ou définitivement perdu leur emploi, et ils ont été contraints de rester à la maison. D'autres ont dû rapidement transformer leurs tâches quotidiennes en télétravail. D'un autre côté, les contextes éducatifs (p. ex. milieu de garde, école) ont également cessé temporairement leurs activités ; les enfants sont restés à la maison avec leurs parents, certains pour quelques semaines, d'autres pour plusieurs mois. Le cumul obligé du télétravail et de la prise en charge à temps plein de leurs jeunes enfants a pu générer un stress considérable, mettant à l'épreuve de nombreux parents dans l'exercice de leur fonction de la parentalité (Baert, Lippens, Moens, Weytjens, \& Sterkens, 2020 ; Couillard, 2020 ; Hamouche, 2020).

Puis, le retour au travail et le souci d'accompagner leurs enfants de la meilleure manière possible a pu amener, pour certains parents, un lot d'incertitudes et de questionnements. Par exemple, au Québec, certains étaient préoccupés par les questions sanitaires et la contagion du virus (Ruel, Harvey, \& Leduc, 2020). Cette peur d'être contaminé a pu réduire la participation des familles avec de jeunes enfants à des activités extérieures, d'autant plus que les aires de jeu dans les parcs étaient fermées pendant plusieurs mois, de même que les terrains de sports, les plages, etc. Ainsi, le contact avec d'autres familles est devenu plus limité et a pu engendrer un important isolement social. Pour plusieurs familles, à cet isolement s'est ajouté l'insécurité alimentaire, le manque de matériel technologique et les obstacles de communication entre l'école et les familles, surtout pour les familles qui ne parlent pas la langue de scolarisation (Chantier montréalais Transition vers l'école \& Ruel, 2020).

\footnotetext{
${ }^{1}$ Partie francophone de la Belgique.

@2021 Duval, Lehrer, Pirard, Housen

DOI https://doi.org/10.51657/ric.v5i1.41066
} 
Revue internationale du CRIRES : innover dans la tradition de Vygotsky (2021), vol 5, no 1

\section{En milieux de garde...}

Plusieurs changements ont aussi été observés dans les milieux de garde. Par exemple, au Québec, des Centres de la petite enfance [CPE] ont été transformés en service d'urgence, où on accueillait uniquement les enfants de travailleurs essentiels (p. ex. domaine de la santé). En raison de la relocalisation des services, ces CPE recevaient des enfants qui ne fréquentaient pas ce milieu de garde initialement. Le personnel éducateur qui travaillait auprès des enfants dont les parents étaient considérés comme des travailleurs essentiels n'était ainsi pas disponible pour organiser et animer des activités de transition avec les enfants du groupe habituel, même à distance. Le contact entre les éducateurs, les enfants et leurs familles ont été restreints, voire rendus impossibles. Pour les nombreux enfants qui fréquentaient un milieu de garde avant leur entrée à l'école, cela a pu entraîner une perte de repères pour eux, leurs familles et les professionnels impliqués dans la transition (Bigras, Laurin, Lemay, Lehrer, Charron, Duval, \& Mazaye-Robert, accepté).

Àl'école...

Du côté des enseignants, la fermeture des écoles, réalisée au printemps 2020, les a contraints à revoir leurs pratiques pédagogiques pendant plusieurs semaines, de même que la mise en place de plusieurs pratiques de transition collaboratives qui sont habituellement vécues dans les milieux éducatifs (p. ex., visite de la classe avant la rentrée par l'enfant et sa famille) (Chantier montréalais Transition vers l'école \& Ruel, 2020). Ainsi, les manières d'accueillir les enfants et leurs parents à l'école ont été revues, et ce, à vitesse grand V. Les éducateurs et les enseignants ont dû user (rapidement) de créativité pour dialoguer avec les parents.

Au Québec toujours, Ruel et ses collaborateurs (2020) ont effectué, au printemps 2020, un sondage auprès des organismes de coordination et de concertation montréalais en petite enfance. Ce sondage, transmis aux 29 territoires montréalais, a été répondu par 16 territoires (taux de participation $=55 \%$ ). Ce sondage visait à connaître la situation sur le terrain montréalais pour pouvoir mieux soutenir les acteurs et garder vivant le lien entre les partenaires locaux et régionaux. La plupart des questionnements relevés par les participants au sondage étaient rattachés aux mesures habituelles de transition qui ont été interrompues (Ruel et al., 2020), puisque les efforts étaient davantage consacrés à ajuster les pratiques actuelles en réponse aux normes sanitaires. Ainsi, dans certaines provinces (p. ex. Ontario, Canada), les enseignants ont dû enseigner à distance, même auprès des enfants d'âge préscolaire. De plus, les activités de transition traditionnelles, telles que les visites en classe, ont été annulées ou transformées en activités virtuelles (p. ex. échanges sur des plateformes telles que Zoom ou Teams, etc.) ou par le partage de différents documents (p. ex., visite à l'aide d'un album photo).

Des constats similaires sont observés dans certains pays et régions de l'Europe. Par exemple, en FW-B, des enseignants ont rapidement adapté leurs pratiques de classe au printemps 2020 , en vue de respecter les «bulles » imposées par le gouvernement. Ainsi, les enfants ${ }^{2}$ qui auparavant mangeaient avec tous les autres écoliers dans un grand réfectoire ont été amenés à prendre leur repas dans leur propre classe. Les temps de récréation ont également été aménagés en vue d'éviter des regroupements trop grands d'enfants. Le nombre d'adultes (pouvant aller jusqu'à

\footnotetext{
${ }^{2}$ En FW-B, les enfants entrent à 2,5 ans dans le système scolaire

@2021 Duval, Lehrer, Pirard, Housen
}

DOI https://doi.org/10.51657/ric.v5i1.41066 
15 personnes différentes en FW-B) prenant en charge les enfants a également été réduit en vue de limiter les contacts. Les aménagements forcés par la pandémie semblent aller vers davantage de continuité physique, organisationnelle et développementale telle que définies par Dockett et Perry (2007). En diminuant le nombre de changements spatiaux que peuvent vivre les enfants et en réduisant le nombre d'adultes œuvrant auprès d'eux, les continuités physique et organisationnelle sont améliorées. Par conséquent, cela permet une plus grande prise en compte de la continuité développementale caractérisée entre autres par la reconnaissance des besoins de repères et de stabilité des enfants dans un contexte où les pratiques de transition sont encore peu développées (Housen et Royen, 2019).

Enfin, tel qu'évoqué précédemment, les transitions peuvent être classées selon leur degré de prévisibilité (Schlossberg, 2005), nous amenant à répertorier trois types de transition. Pour les enseignants, si la transition en soi est anticipée, la crise de la Covid est non anticipée, et donc, le personnel enseignant se trouve dans un contexte qui augmente le poids des incertitudes. La crise a notamment été présente à deux moments importants du vécu scolaire : la fin de l'année scolaire (printemps 2020) et la rentrée scolaire (automne 2020).

\section{Lors de la rentrée scolaire ...}

Si la fin de l'année scolaire a été chamboulée par l'arrivée de la pandémie, la rentrée scolaire de l'automne 2020 a également revêtu des conditions différentes de celles connues auparavant. $\mathrm{Au}$ Canada, la majorité des gouvernements provinciaux ont élaboré différents scénarios avant la rentrée scolaire, afin d'orienter les commissions scolaires dans les actions à implanter. Certaines provinces ont par ailleurs laissé aux commissions la décision de choisir le scénario à appliquer parmi ceux proposés (St-Marie, 2020). Au Québec, il semble que plusieurs questions demeuraient encore floues lors de la rentrée 2020, tel que rapporté par un journaliste (Meloche-Holubowski, 2020, 11 août). Dans un rapport préparé par le Centre de recherche et d'intervention sur la réussite scolaire [CRIRE], basé sur le document Tracking Canada's education systems' response to COVID-19 (People for Education, 2020), il est indiqué que le gouvernement québécois n'avait toujours pas, en date du 24 septembre 2020, partagé un plan d'urgence provincial. En effet, il laissait plutôt ce rôle aux centres de services scolaires, aux commissions scolaires anglophones et aux établissements d'enseignement (St-Marie, 2020). Ainsi, plusieurs aspects concernant les mesures d'urgence (p. ex. adaptation des modalités d'apprentissage, planification de la collaboration avec les familles) devaient être planifiés et organisés par le milieu scolaire, et ce, en fonction de ses besoins.

De façon générale, les enseignants ont dû revoir la manière d'accueillir les enfants et leurs parents, en plus de repenser l'organisation de la classe (p. ex. matériel offert, activités de connaissance en début d'année, etc.) et leurs pratiques pédagogiques (p. ex. proximité physique avec les enfants, etc.). Au Québec, le Gouvernement a dévoilé, en août 2020, les mesures prévues pour l'entrée scolaire : les enfants nouvellement accueillis à la maternelle devaient demeurer ensemble, avec les autres élèves de leur groupe, dans l'idée de former des « bulles », dans le respect des consignes de distanciation déterminées par les autorités de santé publique. Les enseignants de la maternelle (4 et 5 ans) n'avaient pas de distanciation à respecter avec leurs élèves, mais ils devaient porter obligatoirement un équipement de protection individuelle, c'est-à-dire un masque

○2021 Duval, Lehrer, Pirard, Housen 
Revue internationale du CRIRES : innover dans la tradition de Vygotsky (2021), vol 5, no 1

et une visière (Gouvernement du Québec, 2020). Pour les enfants, les directives pour le port du masque variaient en fonction des activités.

Les nouvelles mesures ont pu être déstabilisantes pour certains enfants et leur famille. Par exemple, dans certaines écoles, des enfants ne connaissaient pas leur enseignante attitrée au début de l'année scolaire, comme cela est habituellement le cas. À titre illustratif, dans un article de presse où un journaliste rapportait ses propos tenus avec différents membres du personnel scolaire (Bilodeau, 2020, 23 août), des enseignantes ont déclaré avoir pris trois semaines pour évaluer les enfants et former des classes dites équilibrées. La maman d'un enfant qui commençait l'école rapportait que : «D'habitude, au printemps, l'école organise une journée d'accueil. Les parents font le tour de l'école tandis que les enseignants et les orthopédagogues observent les enfants pendant qu'ils jouent. Ils forment les groupes de cette manière » (Bilodeau, 2020, 23 août). Bref, ces ajustements, notamment rapportés dans les médias, montrent que les milieux scolaires québécois ont inévitablement dû composer avec le contexte imprévisible de la COVID-19 pour une durée indéterminée.

Si des ajustements ont été mis en place dans les provinces canadiennes, notamment au Québec, plusieurs inquiétudes ont aussi été relevées concernant la rentrée scolaire de l'automne 2020 dans certains pays et régions de l'Europe. Dans une des études menées par Housen (en cours) ${ }^{3}$ en FW-B, des enseignantes œuvrant à l'éducation préscolaire ont notamment évoqué qu'elles se préoccuppaient de la manière d'accueillir les parents dans leur classe lors du premier jour de la rentrée scolaire. Ainsi, le cas de cette enseignante manifestant de l'inquiétude face au fait que les enfants n'ont pas pu venir visiter la classe avant leur première rentrée scolaire. Pour tenter d'accompagner ces enfants (et leur famille), l'enseignante a décidé d'envoyer, par la poste, un album avec différentes photos de sa classe, et ce, à ceux qui allaient bientôt faire leur rentrée à l'école. Ce genre d'initiatives propre à chacun des milieux scolaires, voire à chaque enseignante est peu répandu et peut paraître novateur en Belgique francophone. En Écosse, les enseignants ont manifesté, en août 2020, des inquiétudes à l'égard de la rentrée scolaire. En effet, une enquête menée auprès de 30000 enseignants par le syndicat des enseignants de l'Educational Institute of Scotland a révélé que, si $60 \%$ des participants ont exprimé leur soutien à la décision de rouvrir les écoles, $66 \%$ d'entre eux étaient anxieux et ne croyaient pas que des facteurs atténuants à la crise étaient bel et bien mis en place dans leur milieu. Ces données ont été diffusées dans un média électronique, et rapportées par un journaliste (Hepburn, 2020, 10 août).

Quoi qu'il en soit, si des préoccupations sont observées un peu partout à travers le monde, force est de constater que les différents acteurs œuvrant en éducation ont dû, et devront, ajuster leurs pratiques dans l'accueil des enfants à l'école. Et si cette crise devenait un vecteur de changement dans l'éducation à la petite enfance ?

\section{La crise, vecteur de changements ?}

Selon Duterme (2016), tout système a tendance à maintenir relativement stables ses variables essentielles, mais «[...] les crises fournissent dans le secteur public des occasions particulièrement propices au changement [...]» (Rochet, Keramidas, \& Bout, 2008, p.71). Cette

\footnotetext{
${ }^{3}$ Housen mène actuellement une thèse de doctorat sur la transition vers l'école, à l'Université de Liège, en Belgique.
}

@2021 Duval, Lehrer, Pirard, Housen

DOI https://doi.org/10.51657/ric.v5i1.41066 
crise sanitaire, liée à la pandémie de COVID-19, semble avoir entraîné certains changements de pratiques et d'organisation dans la prise en charge des enfants. Sur base des travaux de Watzlawick, Weakland et Fisch (1981), Duterme (2016) distingue deux types de changement : les changements de type 1 sont caractérisés par des modifications internes tout en maintenant l'ensemble du système en place, tandis que les changements de type 2 «[..] modifient une ou plusieurs normes d'équilibrage du système [...], ils sont nécessairement plus difficiles et plus longs à effectuer que des changements de type $1 \gg$ (Duterme, 2016, p. 36). Les changements qui surviennent dans les systèmes peuvent être catégorisés en type 1 et 2 selon qu'ils affectent ou non l'équilibre de l'ensemble du système.

Les premiers constats relevés ci-avant quant aux changements réalisés dans les milieux éducatifs, de façon à soutenir la rentrée vers l'école, semblent indiquer des changements de type 1 relatifs aux pratiques de transition dans les classes. En effet, des acteurs de différents établissements modifient leurs pratiques afin d'être en accord avec les réglementations sanitaires, mais cela n'affecte pas nécessairement de manière conséquente l'ensemble du fonctionnement du système scolaire. Cette forme de résilience organisationnelle dont les acteurs scolaires ont fait preuve et qu'ils devront maintenir lors de la rentrée prochaine peut constituer « un véritable vecteur stratégique de changement dans l'organisation » (Rochet et al., 2008, p. 71). Comment faire de cette crise et des changements observés un momentum pour passer d'un changement organisationnel à un changement institutionnel ? Comment mobiliser l'ensemble du système afin de faire évoluer ces changements de type 1 en changements de type 2 permettant un rééquilibrage institutionnel ? En temps de pandémie, la planification d'une transition de qualité a été perturbée, ce qui a pu nuire au développement de liens de confiance réciproque entre l'enfant, sa famille et l'école, ainsi qu'à la confiance de l'enfant envers ses capacités et à l'engagement du parent. Bien que des initiatives ont été mises en place dans divers milieux et différents pays, de manière à tisser des liens de collaboration entre les acteurs engagés dans la transition vers l'école, que peut-on en retirer?

\section{Un risque ou de nouvelles occasions pour des pratiques de transition et de collaboration?}

En tout temps et particulièrement en période de crise, différentes mesures collaboratives peuvent être mises en place afin de favoriser la continuité entre les milieux fréquentés par l'enfant (Dockett et Perry, 2003). En raison de la pandémie, toutes les familles, et particulièrement celles qui étaient déjà en situation de vulnérabilité, peuvent être soumises à un stress accru. Ainsi, il est particulièrement important lors de cette première rentrée scolaire d'envisager d'utiliser une combinaison d'activités et de moyens (p. ex. ressources en ligne et imprimées et disponibles dans les langues maternelles des familles), et de fournir des documents papier aux familles qui n'ont pas accès à Internet. Outre les familles en situation de vulnérabilité, d'autres particularités familiales sont à considérer pour mieux accompagner chacun des enfants : parents allophones, parents qui travaillent à temps plein (télétravail) et qui s'occupent aussi de leurs enfants à temps plein, familles monoparentales, etc. Les interactions école-famille, de même que les collaborations entre les milieux éducatifs (p. ex. personnel éducateur et enseignant), doivent ainsi être pensées pour agir comme facteur de soutien et de protection nécessaire à la collaboration et non comme des formalités administratives qui risqueraient d'être vécues comme des charges supplémentaires, avec ses effets de normalisation et de contrôle.

○2021 Duval, Lehrer, Pirard, Housen 
Revue internationale du CRIRES : innover dans la tradition de Vygotsky (2021), vol 5, no 1

\section{Occasions de collaboration avec les familles}

Dans un document produit par l'Institut national de santé publique du Québec (INSPQ, 2020), lequel présente des conditions favorables aux transitions scolaires recensées par différents experts tout en soulignant les implications du contexte de la COVID-19, il est proposé de susciter un sentiment d'appartenance chez les familles, en tentant par exemple de poursuivre la tenue d'activités transitionnelles implantées avant, pendant et après le moment de transition, tout en respectant les mesures sanitaires en vigueur. Des travaux suggèrent aussi d'utiliser différentes occasions (ex. : distribution de paniers alimentaires) pour rencontrer les familles (Chantier montréalais Transition vers l'école \& Ruel, 2020) et leur donner du matériel éducatif ou la liste des ressources locales disponibles, en portant une attention accrue aux familles d'enfants ayant des besoins particuliers (Ruel, Moreau, \& April, 2014). De plus, pour assurer un contact avec les enfants et leurs parents, certaines actions ont pu être mises sur pied par différents organismes. Des équipes d'enseignants ont par exemple organisé des visites virtuelles de leur école, ou une visite physique de leur cour d'école, pour établir un lien avec les familles et les enfants (Chantier montréalais Transition vers l'école \& Ruel, 2020). Aussi, un échange de photos par courriel est une pratique de transition qu'il a été possible de mettre en place, malgré la situation de distanciation physique.

Toutefois, plusieurs activités jugées essentielles pour tisser des liens avec la famille ont aussi dû être annulées en raison des normes sanitaires. Par exemple, bien que des enseignantes de maternelle jugent que : "La présence des parents le premier jour de maternelle est essentielle », certaines écoles ont dû annoncer qu'elles n'accueilleraient pas les parents des tout-petits en raison de la COVID-19 lors du premier jour d'école, et ce, tel que rapporté dans les médias (Bilodeau, 2020, 23 août). Pour certaines enseignantes, cela est très désolant, car cette présence parentale est importante pour rassurer les enfants lors de leur rentrée vers d'école, mais cela est aussi très important pour créer un lien avec les parents, pour apprendre à les connaitre. De plus, des travaux ont montré que le stress induit par la pandémie et la crainte de contamination en milieu scolaire a pu diminuer la présence, la disponibilité et la capacité de collaborer de certains acteurs de la communauté (p. ex. milieu scolaire) et des parents (Gupta \& Jawanda, 2020; Imran, Zeshan, \& Pervaiz, 2020).

Selon Ruel, Moreau, Bérubé et April (2015), quatre dimensions sont à prendre en considération lorsque vient le temps de planifier une transition de qualité : 1) Assurer les conditions favorables 2) Soutenir l'engagement du parent 3) Consolider le partenariat entre les services préscolaires 4) Renforcer les pratiques spécifiques pour les enfants à besoins particuliers. Il s'agit entre autres de reconnaitre la place prépondérante des parents, en les mettant à contribution pour présenter leur enfant à l'école, avec ses forces, ses qualités et ses besoins. Ce type de pratiques a pu être observé dans différents milieux, tel que rapporté dans les écrits. Par exemple, aux ÉtatsUnis, la communauté d'Altgeld-Riverdale, située dans la partie sud de Chicago, adopte une approche de collaboration communautaire pour aborder la transition vers la maternelle, comme on peut l'observer aussi dans certains contextes québécois. En règle générale (en temps de « nonpandémie »), des activités de transition ont lieu dans les milieux scolaires, telles que des réunions conjointes entre les enseignants de la maternelle, des soirées d'activités familiales organisées dans les écoles primaires locales et des excursions dans la ville qui rassemblent des élèves de la maternelle de différentes écoles. Les activités de transition qui auraient généralement lieu ont évidemment été annulées en raison de la pandémie. Au lieu de cela, des activités de transition qui

@2021 Duval, Lehrer, Pirard, Housen

DOI https://doi.org/10.51657/ric.v5i1.41066 
sembleraient inhabituelles en temps normal et donc novatrices dans ce contexte non anticipé ont été mises sur pied, tel que rapporté par un analyste politique en éducation et la directrice de la politique d'éducation préscolaire et élémentaire de New America (Loewenberg \& Bornfreund, 2020, 24 juin). Les nouvelles mesures, mises en place en temps de pandémie, ont été les suivantes : des réunions virtuelles permettant aux élèves de rencontrer leurs futurs enseignants de maternelle, des réunions virtuelles pour que les familles puissent discuter avec les enseignants de la maternelle, des « heures du conte» vécues en ligne (enseignants qui racontent une histoire aux enfants de manière virtuelle), etc. (Loewenberg \& Bornfreund, 2020, 24 juin).

En FB-W, des études de cas (Housen, Royen, \& Pirard 2020) ont mis en évidence une adaptation des pratiques de collaboration école-famille au printemps 2020. Ces analyses ont permis, d'une part, de montrer que les enseignantes participantes considèrent aussi que la présence des parents est essentielle lors de la première journée d'école (Housen et al., 2020). Pour tenter de pallier ce défi (soit d'inviter les parents en classe malgré les normes de distanciation en vigueur), une participante a décidé, en accord avec la direction de l'école, de réaliser une rentrée échelonnée pour les enfants et leur famille. Ainsi, elle pouvait accueillir les enfants et leurs parents en classe, car un nombre limité de personnes étaient présentes dans l'environnement à la fois. Pour ce faire, l'enseignante a dû organiser la rentrée et prévoir un horaire pour tous les enfants de sa classe : certains parents pouvaient se présenter à $8 \mathrm{~h} 30$, d'autres à $9 \mathrm{~h} 00$, et ainsi de suite. Bien que ce genre d'initiatives aient été implantées dans certains milieux, cela demeure encore une fois à la discrétion des enseignantes et des écoles.

De plus, toujours en FW-B, plusieurs professionnels ont mentionné, dans les études de cas (Housen et al., 2020), qu'ils avaient tenté de maintenir le contact avec les familles au travers de divers moyens, pour la plupart technologiques, mais aussi en allant apporter du matériel pédagogique (p. ex. ouvrages jeunesse) dans les boîtes aux lettres des familles. Ces nouvelles pratiques ont permis, en quelque sorte, de renforcer les liens de collaboration entre l'école et la maison. En ce sens, une maman témoigne et explique qu'avant la crise de la COVID-19, elle avait très peu de contacts avec l'école :

C'est 30 secondes à 1 minute à l'arrivée ou à la sortie (mais maintenant) [...] je dois dire le truc Teams ${ }^{4}$ c'est assez chouette parce qu'il y autant des mots généraux de la directrice que des mots généraux de l'enseignante ou bien justement on peut avoir des conversations plus en seul à seul avec l'enseignante en posant des questions. Moi je trouve que c'est beaucoup plus chouette en tout cas on a un contact beaucoup plus rapproché quoi (Housen et al., 2020).

Ces contacts plus rapprochés, mais aussi pouvant être initiés par le parent, semblent davantage satisfaire les besoins de cette maman. Bien que l'ensemble de ces initiatives ont permis des moments d'échanges virtuels, ont-elles renforcé la collaboration entre les différents acteurs impliqués dans la transition vers l'école ? Est-ce que le rôle du parent est bel et bien reconnu dans la transition scolaire ? Bien que la collaboration école-famille ait pu être possible, voire même améliorée à distance (p. ex. appels Skype, conversations téléphoniques, etc.), des lacunes sont également observées. Par exemple, lorsque les enfants sont retournés en classe au printemps 2020, les parents ne pouvaient plus rentrer dans l'école, même pour les nouveaux enfants qui réalisaient

○2021 Duval, Lehrer, Pirard, Housen 
Revue internationale du CRIRES : innover dans la tradition de Vygotsky (2021), vol 5, no 1

leur premier jour à l'école ${ }^{5}$. Bien qu'il y ait des injonctions et une volonté de collaboration dans les discours « officiels» des acteurs scolaires, dans les faits il semble y avoir de nombreuses résistances (Conus \& Ogay, 2018).

\section{Occasions de collaboration entre les acteurs concernés par la transition}

Comme le suggèrent Ruel et ses collaborateurs (2015) dans leur troisième dimension pour planifier une transition de qualité, différentes initiatives régionales ont été implantées au printemps, afin d'établir une collaboration entre les acteurs concernés par la transition. À titre illustratif, au Québec, la collaboration entre différentes organisations impliquées dans la concertation intersectorielle Main dans la Main ${ }^{6}$ s'est poursuivie malgré les mesures de confinement mises en place au printemps, et les actions qui avaient initialement été planifiées par les partenaires (p. ex. milieu de garde, école, organismes communautaires) ont été ajustées. Ces derniers ont réussi à maintenir la communication entre eux, et ainsi connaitre les changements apportés au sein des différents milieux éducatifs. De plus, toujours au Québec, le déploiement d'agents de transition scolaire, initiative découlant de la Stratégie 0-8 an ${ }^{7}$, a aussi permis de poursuivre les collaborations entre les partenaires (p. ex. milieu de garde et école) durant le contexte de pandémie. En effet, le mandat des agents de transition, qui œuvre sur des territoires précis, consiste à promouvoir et de développer des partenariats impliquant les acteurs clés qui participeront à l'instance de concertation territoriale et d'animer les rencontres permettant des échanges au sujet des priorités et préoccupations de ces acteurs en matière d'interventions transitionnelles (Groupe de travail régional en transition scolaire, 2018).

\section{Occasions de soutenir les enfants ayant des besoins particuliers}

La quatrième dimension permettant de planifier une transition de qualité, selon Ruel et ses collaborateurs (2015) concerne la mise en place de pratiques spécifiques de transition en lien avec les enfants ayant des besoins particuliers. Pour ce faire, il est suggéré de planifier la rentrée avec tous les acteurs qui connaissent l'enfant et prévoir la mise en place des services requis avant la rentrée. Toutefois, dans le contexte actuel, cela semble plutôt ardu à mettre en place, et des lacunes sont également observées. Par exemple, au Québec, le ministère de l'Éducation et des études supérieures (2020) a prévu un budget pour engager plus de spécialistes pour soutenir les enfants en difficulté dans les classes. Or, cette annonce est survenue en août, pratiquement une semaine avant la rentrée scolaire. Comment peut-on penser que la continuité des services est assurée dans cette urgence d'agir?

\footnotetext{
${ }^{5}$ En FW-B, l'enfant peut débuter l'école maternelle à différents moments de l'année, et ce, pas nécessairement au mois de septembre. La date de rentrée est alors décidée selon la date de naissance de l'enfant. Certains enfants ont dû transiter vers l'école au printemps 2020, soit au cœur même de la pandémie.

${ }^{6}$ Cinq partenaires sont liés au projet de concertation Main dans la main : Commun'action 0-5, Regroupement des Centres de la Petite Enfance des Régions de Québec \& Chaudière Appalaches [RCPECQ], l'Initiative 1, 2, 3, Go ! Limoilou, la Direction de la santé publique ainsi que le centre de services scolaire de la Capitale.

${ }^{7}$ Mise en place par le MÉES, cette Stratégie (aussi appelée Tout pour nos enfants) constitue un jalon important de la Politique de la réussite éducative, qui vise à soutenir les Québécoises et les Québécois dans leurs apprentissages, dès leur petite enfance. Voir ce lien pour davantage d'informations : https://securise.education.gouv.qc.ca/politique-de-lareussite-educative/toutpour-nos-enfants/.
}

@2021 Duval, Lehrer, Pirard, Housen

DOI https://doi.org/10.51657/ric.v5i1.41066 


\section{Occasions de mettre en auvre une démarche concertée, planifiée et structurée}

L'une des dimensions permettant de mettre en place des conditions gagnantes pour une transition de qualité vise à mettre en œuvre une démarche concertée, planifiée et structurée, en prévoyant du temps à la planification de la transition. Pour ce faire, des conditions doivent être mises en place à cet égard : temps de libération (ou de concertation) pour le personnel enseignant, accompagnement d'un agent de transition, etc. (Ruel, 2020). De plus, le soutien de la direction d'école est primordial pour que les enseignants puissent bénéficier de conditions gagnantes pour planifier la transition vers l'école. Cependant, encore une fois, cette dimension semble difficile à considérer dans le contexte actuel.

Par exemple, au Québec, il y a actuellement une pénurie d'enseignants, et plusieurs articles de presse ont été publiés en ce sens. À titre illustratif, la journaliste Marie-Ève Morasse écrivait, en août 2020, que dans la région de Montréal, l'Alliance des professeures et des professeurs de Montréal estimait à 160 le nombre de postes d'enseignants qui restaient à pourvoir, tandis que le syndicat affirmait plutôt un manque d'environ 500 enseignants dans les classes pour la rentrée scolaire (Morasse, 2020, 20 août). Le réseau de l'éducation québécois est donc soumis à une pression importante pour trouver du personnel enseignant, et les directions d'école s'en inquiètent. "Il y a une pénurie dans toutes les sphères. Chez les enseignants, chez les professionnels, chez les directions d'école et, le plus dramatique, chez les éducatrices en service de garde [...] » (Morasse, 2020, 20 août). D'ailleurs, au début de l'année 2020, le ministre JeanFrançois Roberge a estimé que la pénurie de main-d'œuvre dans les écoles du Québec serait son plus grand défi et qu'il faudrait «quelques années» pour y remédier. Pour sa part, et tel que rapporté par une journaliste de la Presse canadienne, le ministre Roberge n'a pas chiffré la pénurie d'enseignants, mais en septembre 2019, son ministère avait calculé qu'il manquait au moins 360 enseignants pour combler les besoins dans les écoles du Québec (Plante, 2020, 14 janvier).

En temps de pandémie, ce défi (pénurie d'enseignants) a pu amener plusieurs ajustements supplémentaires, voire des conséquences sur le personnel enseignant et les enfants. Par exemple, les enseignants n'ont pas nécessairement eu le temps pour être libérés, car il n'y avait aucune ressource disponible pour les remplacer dans les classes, et parce qu'ils devaient plutôt veiller, en début d'année scolaire, à ce que leur classe soit désinfectée, à acheter les produits sanitaires demandés, etc. Ils manquaient ainsi de temps pour discuter entre eux, voire pour planifier et organiser les activités de transition. Or, tel que mentionné plus tôt, il importe de se soucier de la continuité entre les milieux fréquentés par l'enfant, afin de développer une vision partagée de la transition et de minimiser les discontinuités qui peuvent survenir entre les services éducatifs. Des travaux ont par ailleurs documenté des effets de la crise de COVID-19 sur les activités favorisant la continuité ; par exemple, la fermeture soudaine des milieux de vie précédents (p. ex. fermeture des services de garde éducatifs au printemps 2020) a pu créer une discontinuité entre les milieux éducatifs (p. ex. perte de routines, impossibilité de visiter le futur milieu éducatif, difficulté à entretenir la création de partenariats, etc.) (Coddind, Collier-Meek, Jimerson, Klingbeil, Mayer, \& Miller, 2020). De plus, des travaux (p. ex. Stratford, 2020, cité dans INSPQ, 2020) ont montré que les temps d'échanges entre les membres du personnel scolaire ont davantage porté sur les mesures de protection à mettre en place en temps de crise, et moins sur le partage de savoirs pour accompagner les transitions scolaires, et donc assurer une continuité. En somme, les moments d'échanges et de concertation entre les acteurs qui gravitent autour de l'enfant, qui sont parfois

@2021 Duval, Lehrer, Pirard, Housen 
Revue internationale du CRIRES : innover dans la tradition de Vygotsky (2021), vol 5, no 1

quasi inexistants dans un contexte « normal », ont été encore plus difficiles à mettre en place dans le contexte de la COVID-19.

De plus, lors de la rentrée scolaire 2020, certains intervenants jugeaient primordial de reprendre la matière manquée au printemps, en raison du confinement. Effectivement, à la mi-août 2020, de nombreux intervenants demandaient au gouvernement de préciser rapidement les mesures prévues pour aider les élèves à combler leur retard scolaire, puisque plusieurs n'avaient pas mis les pieds à l'école depuis la mi-mars (Dion-Viens, 2020, 10 août). On peut se questionner sur cet aspect en lien avec l'entrée à l'école maternelle : Est-ce que des activités plus scolarisantes seront organisées à l'automne 2020, afin de rendre les enfants « prêts » à transiter ensuite vers la première année du primaire ? Est-ce que le personnel enseignant perdra de vue l'importance de l'approche développementale à l'éducation préscolaire (voire même au premier cycle du primaire) ? Puisque le mandat de l'école maternelle consiste notamment à donner le gout de l'école aux enfants et de veiller au bien-être des enfants, il semble essentiel de poser cette question. Il importe d'autant plus de rappeler que la fréquentation de la maternelle n'est pas obligatoire, ni celle dans les services de garde éducatif à l'enfant; alors les enfants n'ont rien manqué.

En FW-B, les temps de concertation qui ont habituellement lieu entre les enseignants, lesquels se réunissent deux heures par semaine pour parler des problématiques vécues en classe, portent habituellement sur les didactiques (p. ex. lecture, numératie, etc.), et non sur la continuité de développement telle que présentée plus tôt dans cet article. Les concertations avec les autres acteurs comme ceux de l'accueil extrascolaire ou de l'accueil de la petite enfance font largement défaut. Les pratiques de transition très peu développées en temps ordinaires selon un état de lieux réalisés sur un échantillon représentatif d'établissements (Housen \& Royen, 2019) occupent une place périphérique dans les projets d'établissement. Toutefois, l'analyse de cas jugés novateurs comparativement aux autres de cette région montre la possibilité d'actions alternatives développées en équipe avec le soutien des directions (Housen et al., 2020). Dans ce contexte de pandémie qui renforce encore les incertitudes, il semble encore plus essentiel de reconnaître l'importance du soutien des collègues, de la direction, de l'institution et de la gouvernance pour réassurer ces professionnels de l'éducation à la petite enfance dans leur activité quotidienne d'une complexité trop souvent méconnue.

Bref, on peut constater la présence de plusieurs occasions pour déployer des mesures permettant de faciliter la transition vers l'école, mais aussi plusieurs défis, notamment en cette période de pandémie.

\section{Conclusion}

La transition vers l'école vécue en contexte de pandémie a amené les différents acteurs impliqués à planifier une situation connue et anticipée (entrée vers l'école) dans un contexte où l'imprévisibilité était bien présente. La concertation entre ces derniers est reconnue comme un élément clé pour assurer une continuité entre les environnements (familial et éducatif) fréquentés par l'enfant. Une préparation adéquate s'impose de la part de tous les membres du milieu scolaire (p. ex. personnel éducateur et enseignant), ainsi que de l'enfant et sa famille, pour soutenir la transition vers l'école et les ajustements qu'elle implique (Dockett \& Perry, 2009 ; Jacques \& Deslandes, 2002). Pour cause, des liens peuvent être tissés entre ces effets relevés sur l'enfant en 
période de pandémie et les enfants qui ont vécu une guerre ou une autre crise imprévue dans leur vie (p. ex. tornade, inondation, mort d'un parent, etc.). En effet, la pandémie représente une période de crise qui doit être considérée dans le développement et l'apprentissage des enfants.

Rappelons que les jeunes enfants sont particulièrement vulnérables face aux catastrophes et aux situations de crise, lesquelles peuvent avoir des effets à court et à long terme sur leur fonctionnement psychologique et socioémotionnel, de même que sur leur santé générale (Schonfeld et al., 2020). C'est pourquoi plusieurs travaux ont montré l'importance d'une stratégie globale d'intervention (Palluy, Choinière, \& Roberge, 2010; Statford, Cook, Hanneke, Katz, Seok, Steed, Fulks, Lessans, \& Temkin, 2020), qui renvoie à la mise en place d'un climat scolaire bienveillant et sécuritaire, tant pour les enfants que pour le personnel scolaire. Quels seront les effets à court et long terme de la pandémie sur les jeunes enfants ? Quelles mesures seront mises en place après la crise afin de bien soutenir ces enfants qui ont débuté l'école dans une situation étrange et insécure ? Mais aussi, comment « profiter » de ce moment pour transformer un système, pour en faire des changements profitables à la mise en place de pratiques de transition collaboratives?

Selon la manière dont elle est comprise, gérée dans l'institution scolaire, on peut se demander si la pandémie va contraindre encore davantage ou au contraire favoriser la mise en place et le développement de pratiques de collaboration entre l'école et la famille dès la première transition. Est-ce que des pratiques de collaboration misant sur la continuité pourraient être davantage observées dans les milieux éducatifs ? Est-ce que la crise de la COVID-19 pourrait mener vers des pratiques de collaboration positives et durables entre les différentes institutions liées au monde de la petite enfance ? Est-ce que la pandémie peut représenter un vecteur de changement en ce qui concerne les pratiques de collaboration entre l'école et la famille ? Voilà plusieurs questions qui semblent nécessaires d'évoquer, bien que les réponses ne puissent pas être fournies dans ces moments d'incertitude.

Les enseignants œuvrant à l'école maternelle doivent se rappeler qu'ils peuvent influencer positivement et durablement les capacités d'adaptation de l'enfant, en mettant en place des pratiques de collaboration positives et harmonieuses avec la famille. Il importe notamment de miser sur la continuité entre les expériences vécues par l'enfant. Puisque les transitions s'inscrivent à long terme dans le parcours de vie de la personne, il est primordial de mettre en place des pratiques visant à favoriser le bien-être émotionnel des enfants, en misant notamment sur l'empathie et la sensibilité. Enfin, dès qu'il le sera possible, il serait profitable d'ouvrir toutes grandes les portes de l'école et de repenser les pratiques de distanciation traditionnelles entre école et familles. Il s'agit ainsi de se mettre en garde contre l'enracinement de certaines pratiques, comme le fait que les parents ne peuvent plus entrer dans les écoles. Si chacun parvient à développer les collaborations indispensables à une transition en douceur particulièrement cruciale en période de crise, peut-être pourrons-nous observer non seulement des changements de surface, mais aussi une véritable métanoïa même dans les différents contextes, qu'ils soient novateurs ou en émergence. 
Revue internationale du CRIRES : innover dans la tradition de Vygotsky (2021), vol 5, no 1

\section{Références}

Ackesjö, H. (2014). Children's transitions to school in a changing educational landscape: borders, identities and (dis-) continuities. International journal of transitions in childhood, 7, 3-15. https://www.diva-portal.org/smash/record.jsf?pid=diva2\%3A795702\&dswid=3759

Ahtola, A., Björn, P. M., Turunen, T., Poikonen, P. L., Kontoniemi, M., Lerkkanen, M. K., \& Nurmi, J. E. (2016). The concordance between teachers' and parents' perceptions of school transition practices: A solid base for the future. Scandinavian Journal of Educational Research, 60(2), 168-181. https://doi.org/10.1080/00313831.2014.996598

Baert, S., Lippens, L., Moens, E., Weytjens, J., \& Sterkens, P. (2020). The Covid-19 Crisis and Telework: A Research Survey on Experiences, Expectations and Hopes. IZA Discussion Paper No. 13229. https://ssrn.com/abstract $=3596696$

Ballam, N., Perry, B., \& Garpelin, A. (2017). International perspectives on the pedagogies of educational transitions. Dans N. Ballam, B. Perry, \& Garpelin (dir.), Pedagogies of Educational Transitions (p. 1-12). Springer. https://link.springer.com/chapter/10.1007/978-3-319-43118-5_1

Bérubé, A., Ruel, J., April, J. \& Moreau, A. C. (2018). Family preparation for school entry and the role of transition practices. The Journal of Educational Research, 111(4), 398-403. https://doi.org/10.1080/00220671.2017.1284039

Bigras, N., Laurin, I., Lemay, L., Lehrer, J., Charron, A., Duval, S., \& Mazaye-Robert, C. (accepté). Early childhood educators' perceptions of their emotional state, relations with parents, challenges and opportunities during the early stage of the pandemic. Early Childhood Education Journal.

Bilodeau, É. (2020, 23 août). Maternelle: une rentrée « spéciale » pour les parents et les élèves. La Presse. https://www.lapresse.ca/actualites/education/2020-08-23/maternelle-unerentree-speciale-pour-les-parents-et-les-eleves.php

Broström, L. (2019). Childrens view on their learning in preschool and school - reflections and influence on practice. Dans S. Dockett, J. Einarsdottir et B. Perry (dir.), Listening to Children's Advice about Starting School and School Age Care (p. 84-98). Routledge. https://www.routledge.com/Listening-to-Childrens-Advice-about-Starting-School-andSchool-Age-Care/Dockett-Einarsdottir-Perry/p/book/9780815352440

Cantin, G. (2005). Représentation des futures éducatrices en services de garde à l'enfance à l'égard de la relation avec les parents. Thèse de doctorat. Québec : Université de Montréal.

Centre de transfert pour la réussite éducative du Québec (CTREQ). (2018). Les transitions scolaires de la petite enfance à l'âge adulte. http://rire.ctreq.qc.ca/wpcontent/uploads/2018/08/CTREQ-Projet-Savoir-Transitions-scolaires.pdf

Chantier montréalais transition vers l'école et Ruel, J. (2020). Première transition scolaire dans un contexte de crise: un soutien nécessaire auprès des familles. https://www.reseaureussitemontreal.ca/dossiers-thematiques/covid-19-et-reussiteeducative/premiere-transition-scolaire-dans-un-contexte-de-crise/

Coddind, R. S., Collier-Meek, S., Jimerson, D. A., Klingbeil, Mayer, M. J., \& Miller, F. (2020). School Psychology reflections on COVID-19, antiracism, and gender and racial disparities in publishing. School Psychology, 35(4), 227-232.

Conseil supérieur de l'éducation. (2012). Mieux accueillir et éduquer les enfants d'âge préscolaire, une triple question d'accès, de qualité et de continuité des services (Avis au

@2021 Duval, Lehrer, Pirard, Housen 
ministre de l'Éducation, du Loisir et du Sport). www.cse.gouv.qc.ca/fichiers/ documents/publications/Avis/50-0477.pdf

Conus, X. (2015). Devenir parent d'élève : scolarisation des pratiques éducatives parentales et enjeux de reconnaissance. Petite enfance : socialisation et transitions. France : Villetaneuse. hal- 01257976

Conus, X. (2017). Construction du rôle de parent d'élève en Suisse : le territoire familial, une annexe de l'école ? Dans S. Rayna \& P. Garnier (dir.), Transitions dans la petite enfance. Recherches en Europe et au Québec. Peter Lang. https://www.peterlang.com/view/title/63363

Conus, X. \& Ogay, T. (2018). Quand l'enseignant s'imagine collaborer avec le parent. Étude de cas autour de la confiance. La revue internationale de l'éducation familiale, 44(2), p. 4565. https://www.cairn.info/revue-la-revue-internationale-de-l-education-familiale-2018-2page-

45.htm\#: :text=Il\%20montre\%20que\%20pour\%20un,r\%C3\%A9ciproque $\% 2 \mathrm{C} \% 20$ engen dre $\% 201 \mathrm{e} \% 20$ risque $\% 20 \mathrm{~d}$

Cotnoir, M.-J. (2015). Évaluation d'une stratégie pour faciliter la transition scolaire des enfants à la maternelle : l'outil Mon portrait de Magog (Mémoire de maîtrise). Sherbrooke, Canada : Université de Sherbrooke. Repéré à https://savoirs.usherbrooke.ca/bitstream/handle/11143/6768/ Cotnoir_Marie_Josee_MA_2015.pdf?sequence $=3 \&$ isAllowed $=\mathrm{y}$

Couillard, K. (2020). COVID-19 : effets de la crise sur les familles et le développement des toutpetits. Montréal, Canada : Observatoire des tout-petits. https://toutpetits.org/publications/sur-le-radar/covid-19/covid-19-quels-effets-sur-les-familles-et-ledeveloppement-des-tout-petits/

Cowan, P. A. (1991). Individual and family life transitions: A proposal for a new definition. Dans P. A. Cowan \& M. Hetherington (dir.), Family transitions (p. 3-30). Mahwah, NJ : Lawrence Erlbaum.

Curchod-Ruedl, D. \& Chessex-Viguet, C. (2012). De la famille au cycle initial de la société. Rupture ou transition? Dans P. Curchod, P.-A. Doudin, \& L. Lafortune (dir.), Les transitions à l'école (p. 11-31). Presses de l'Université du Québec. https://www.puq.ca/catalogue/livres/les-transitions-ecole-1903.html

Dahlberg, G., Moss, P., \& Pence, A. (2013). Beyond quality in early childhood education and care: Languages of evaluation ( $3^{\text {rd }}$ ed.). Londres, Angleterre: Routledge.

Dechêne, A. \& Dupont, B. (2019). Crises singulières, crise plurielle. Un concept, son évolution et son potentiel pour les humaines. Méthodes et Interdisciplinarité en Sciences Humaines (MethIS), 6, p. 1-8. https://orbi.uliege.be/bitstream/2268/241009/1/PoPuPS\%c2\%a0_\%c2\%a0Crises\%20singu li $\%$ c3\%a8res\%2c\%20crise \%20plurielle.pdf

Deslandes, R. (2013). La collaboration parent-enseignante au regard de l'état actuel des connaissances. Revue préscolaire 51(4), 11-14.

Deslandes, R. et Jacques, M. (2004). Relations famille-école et l'ajustement du comportement socioscolaire de l'enfant à l'éducation préscolaire. Éducation et Francophonie XXXII(1), 172-200.

Deslandes, R., Bastien, N., \& Lemieux, A. (2004). Les conditions nécessaires à une collaboration entre la famille, l'école et la communauté. Vie pédagogique, 133, 41-44. 
Revue internationale du CRIRES : innover dans la tradition de Vygotsky (2021), vol 5, no 1

Dion-Viens, D. (2020, 10 août). Rattrapage scolaire: «la mise à niveau des jeunes, c'est important». TVA Nouvelles. https:/www.tvanouvelles.ca/2020/08/10/rattrapage-scolairela-mise-a-niveau-des-jeunes-cest-important

Di Santo, A., \& Berman, R. (2012). Beyond the preschool years: Children's perceptions about starting kindergarten. Children \& Society, 26, 469-479. https://doi.org/10.1111/j.10990860.2011.00360.x

Dockett, S., Griebel, W., \& Perry, B. (2017). Transition to school: A family affair. In Families and transition to school. Dans S. Dockett, W. Griebel, \& B. Perry (dir.), Families and transition to school (Vol. 21) (p. 1-18). Springer.

https://www.springer.com/gp/book/9783319583273

Dockett, S. \& Einarsdóttir, J. (2017). Continuity and change as children start school. Dans N. Ballam, B. Perry, \& Garpelin (dir.), Pedagogies of Educational Transitions (p. 133-150). Springer. https://link.springer.com/chapter/10.1007/978-3-319-43118-5_9

Dockett, S. \& Perry, B. (2001). Starting School: Effective Transitions. Early Childhood Research \& Practice, 3(2). https://ecrp.illinois.edu/v3n2/dockett.htm1

Dockett, S., \& Perry, B. (2007). Transitions to school: Perceptions, expectations, experiences. UNSW Press.

Dockett, S. \& Perry, B. (2009). Readiness for school: A relational construct. Australian Journal of Early Childhood, 34(1), 20-26.

https://researchoutput.csu.edu.au/ws/portalfiles/portal/8698022

Dockett, S. \& Perry, B. (2015). Transition to School: Times of Opportunity, Expectation, Aspiration, and Entitlement. Dans J. Iorio et al. (dir.), Rethinking Readiness in Early Childhood Education. Implications for Policy and Practice (p. 123-139). Palgrave Macmillan. https:/www.palgrave.com/gp/book/9781137485113

Dunlop, AW. (1998). Assessment as part of a continuity study. Early Years, 19(1), 39-49. https://doi.org/10.1080/0957514980190106

Dunlop, A.-W. (2018). Transitions in Early Childhood Education. Oxford Bibliographies in Education.

https://pure.strath.ac.uk/ws/portalfiles/portal/83533831/Dunlop_2018_Transitions_in_earl y childhood_education.pdf

Duterme, C. (2016). Niveaux d'organisation, niveaux de changement. Communications et management, (1)13, p. 93-111. https://www.cairn.info/revue-communication-etmanagement-2016-1-page-93.html

Duval, S. et Lehrer, J. (sous presse). Les transitions vécues par l'enfant et sa famille à l'éducation préscolaire. Dans A. Charron, J. Lehrer, M. Boudreau et E. Jacob (dir.), L'éducation préscolaire au Québec : fondements théoriques et pédagogiques. Presses Universitaires du Québec.

Einarsdottir, J., Dockett, S., \& Perry, B. (2009). Making meaning: Children's perspectives expressed through drawings. Early Child Development and Care, 179, 217-232. https://doi.org/10.1080/03004430802666999

Fabian, H. (2002). Empowering children for transitions. Dans H. Fabian \& A-W. Dunlop, (dir.), Transitions in the Early Years: Debating continuity and progression for young children in early education (p. 123-134). Routledge.

https://www.taylorfrancis.com/books/e/9780203519851

@2021 Duval, Lehrer, Pirard, Housen

DOI https://doi.org/10.51657/ric.v5i1.41066 
Fabian, H. \& Dunlop, A.-W. (2007). Outcomes of good practice in transitions processes for children entering primary school. Working paper in education 42, Bernard van Leer Foundation. https://files.eric.ed.gov/fulltext/ED522698.pdf

Ferry, J.-M. (2014). Approches différentielles de la notion de crise. Revue économique et sociale : bulletin de la Société d'Études Économiques et Sociales, 72(3-4), 44-54. https://www.revue-res.ch/accueil.html

Garnier, P. \& Brougère, G. (2017). Des tout-petits « peu performants » en maternelle. Ambition et misère d'une scolarisation précoce. Revue française des affaires sociales, , 83-102. https://doi.org/10.3917/rfas.172.0083

Giallo, R., Kienhuis, M., Treyvaud, K., \& Matthews, J. (2008). A psychometric evaluation of the parent self-efficacy in managing the transition to school scale. Australian Journal of Educational and Developmental Psychology, 8, 36-48. https://www.newcastle.edu.au/_data/assets/pdf_file/0008/100331/v8-Giallo-et-al.pdf

Gouvernement du Québec. (2020). À l'école, je me protège et je protège les autres ! https://cdncontenu.quebec.ca/cdn-contenu/adm/min/education/publications-adm/covid-19/affichetteport-du-masque.pdf? 1597860241

Groupe de travail régional en transition scolaire (2018). Cadre de référence montérégien pour soutenir une première transition harmonieuse vers l'école. Une vision partagée et des pratiques concertées pour une entrée scolaire de qualité. Direction de santé publique de la Montérégie. http://extranet.santemonteregie.qc.ca/userfiles/file/santepublique/promotion-prevention/Cadre-reference-monteregien-Premiere-transitionscolaire.pdf

Gupta, S. \& Jawanda, M. K. (2020). The impacts of COVID-19 on children. Acta Paediatrica, 109, 2181-2183. https://doi.org/10.1111/apa.15484

Hamouche, S. (2020). COVID-19 and employees' mental health: stressors, moderators and agenda for organizational actions. Emerald Open Research, 2(15). doi : 10.35241/emeraldopenres.13550.1

Hepburn, H. (2020, 10 août). Most teachers want mandatory face coverings in class. TES Reporter. https://www.tes.com/news/most-teachers-want-mandatory-face-coverings-class

Hopps, K. (2014). ECCE setting + school + communication = What for educator relationships? Early Years: An international Research Journal, 34(4), 405-419. https://doi.org/10.1080/09575146.2014.963032

Housen, M. \& Royen, E. (2019). Les pratiques de transition lors de l'entrée à l'école maternelle : état des lieux (sous la direction scientifique de Pirard, F). Belgique : Université de Liège. hdl.handle.net/2268/252317

Housen, M, Royen, E. \&, Pirard, F. (en collaboration avec Godechard, S., Wuidard, E. \&, Al Jammal, L.). (2020). Les pratiques de transition lors de l'entrée à l'école maternelle : études de cas (sous la direction scientifique de Pirard, F). Belgique : Université de Liège. http://hdl.handle.net/2268/252677

Hughes, C., Daly, I., Foley, S., White, N., \& Devine, R. T. (2015). Measuring the foundations of school readiness: Introducing a new questionnaire for teachers-The Brief Early Skills and Support Index (BESSI). British Journal of Educational Psychology, 85(3), 332-356. https://doi.org/10.1111/bjep.1207

Imran, N., Zeshan, M., \& Pervaiz, Z. (2020). Mental health considerations for children \& adolescents in COVID-19 Pandemic ». Pakistan Journal of Medical Sciences, 36,(COVID19-S4). https://doi.org/10.12669/pjms.36.COVID19-S4.2759.

@2021 Duval, Lehrer, Pirard, Housen 
Revue internationale du CRIRES : innover dans la tradition de Vygotsky (2021), vol 5, no 1

Institut national de santé publique du Québec (INSPQ, 2020). Retour à l'école en temps de COVID-19 : focus sur les transitions scolaires. Québec, Canada : INSPQ.

https://www.inspq.qc.ca/sites/default/files/publications/3058-retour-ecole-transitionsscolaires-covid19.pdf

Jacques, M. et Deslandes, R. (2002). Transition à la maternelle et relations école-famille. Comprendre la famille. Dans C. Lacharité et G. Pronovost (dir.), Actes du 6e symposium québécois de recherche sur la famille (p. 247-260). Québec, Canada : Presses de l'Université du Québec.

Kraft-Sayre, M. E. \& Pianta, R. C. (2000). Enhancing the Transition to Kindergarten: Linking Children, Families, \& Schools. National Center for Early Development \& Learning Kindergarten Transition Studies, University of Virginia. http://plc.dadeschools.net/pdf/ready_Kg-parentHdbk.pdf

Loewenberg, A. \& Bornfreund, L. (2020, 24 juin). Supporting Smooth Transitions into Kindergarten During the COVID-19 Pandemic. New America. https://www.newamerica.org/education-policy/edcentral/supporting-smooth-transitionskindergarten-during-covid-19-pandemic/

Malsch, A. M., Green, B. L., \& Kothari, B. H. (2011). Understanding Parents' Perspectives on the Transition to Kindergarten : What Early Childhood Settings and Schools Can Do for At Risk Families. Best Practice In Mental Health, 7(1), 47-66. https://www.ingentaconnect.com/content/follmer/bpmh/2011/00000007/00000001/art000 04

Marinova, K., Dumais, C., \& Drainville, R. (2020). L'urgence de jouer. Revue préscolaire, horssérie, 1-12.

https://drive.google.com/file/d/1--N8LK97W-1A8cxmAirlj5JRN1gF6_7/view?fbclid=IwAR2mienb0Oc2BEbM3pHDcYAi_0ulUjXvzlfT7wRnBa1QQHQHD Q5-sQRIV3U

McCail, G. (1996). Curriculum continuity between Lothian nursery and early school education. Archived Interim Research Report. Moray House Institute of Education, Heriot Watt Univ.

Ministère de l'Éducation du Québec (2003). Passe partout. Un soutien à la compétence parentale. Prendre le virage du succès. Québec, Canada : Gouvernement du Québec. http://www.education.gouv.qc.ca/fileadmin/site_web/documents/dpse/formation_jeunes/P asse-Partout_s.pdf

Ministère de l'Éducation, du Loisir et du Sport [MELS] (2010). Guide pour une $1^{\text {re }}$ transition scolaire de qualité: services de garde et école. Gouvernement du Québec.

https://www.mfa.gouv.qc.ca/fr/publication/Documents/GuideSoutenirPremiereTransScol Qualite_f.pdf

Moll, L.C., Amanti, C., Neff, D., \& Gonzalez, N. (1992). Funds of Knowledge for Teaching: Using a Qualitative Approach to Connect Homes and Classrooms. Theory Into Practice 31(2), 132-141. https://www.jstor.org/stable/1476399

Morasse, M.- È. (2020, 20 août). Rentrée scolaire: il manque de profs à Montréal. La presse. https://www.lapresse.ca/actualites/education/2020-08-20/rentree-scolaire-il-manque-deprofs-a-montreal.php

@2021 Duval, Lehrer, Pirard, Housen 
Meloche-Hulobowski, M. (2020, 11 août). Le plan pour la rentrée scolaire au Québec accueilli favorablement. Radio-Canada. https://ici.radio-canada.ca/nouvelle/1725631/coronavirusrentree-scolaire-ecoles-reaction-plan-covid-pandemie

Ministère de l'Éducation et des études supérieures (2020). Directives spécifiques pour le milieu de l'éducation (COVID-19). Québec, Canada : Gouvernement du Québec. https://www.quebec.ca/education/organisation-activites-scolaires-covid-19/

National Association for the Education of Young Children. (2020). NAEYC COVID 19 statement. https://www.naeyc.org/ resources/blog/naeyc-COVID-19-statement.

Organisation de coopération et de développement économiques. (2017). Résumé des principaux résultats de "Petite enfance, grands défis 2017 : Indicateurs clés de l'OCDE sur l'éducation et l'accueil des jeunes enfants ». Publication de l'OCDE. http://www.oecd.org/fr/education/scolaire/Resume-des-principaux-resultats-de-petiteenfance-grands-defis-2017.pdf

Organisation de coopération et de développement économiques. (2018). Petite enfance, grands défis $V$. Publications de l'OCDE. https://doi.org/10.1787/9789264300620-fr

Palluy, L., Choinière, M., \& Roberge, M. -C. (2010). Réussite éducative, santé, bien-être : agir efficacement en contexte scolaire: synthèse de recommandations. Montréal, Canada : Institut national de santé publique du Québec. https://www.inspq.qc.ca/publications/1065.pdf

Plante, C. (2020, 14 janvier). La pénurie d'enseignants, le plus grand défi de 2020, selon le ministre Roberge. La presse canadienne. https://www.lesoleil.com/actualite/la-penuriedenseignants-le-plus-grand-defi-de-2020-selon-le-ministre-roberge25b91f7756be78567de1f7f27065e7f4

People for Education (2020). Tracking Canada's education systems' response to COVID-19. Toronto, Ontario: People for Education. https://peopleforeducation.ca/wpcontent/uploads/2020/10/PFE-COVID-Tracker-January-25-2021

Petrakos, H. H., \& Lehrer, J. S. (2011). Parents' and teachers' perceptions of transition practices in kindergarten. Exceptionality Education International, 21(2-3), 62-73.

Lehrer, J., Bigras. N., Charron, A et Laurin, I. (à paraitre), La recherche) en éducation à la petite enfance : origines, méthodes et applications, Québec : Presses de l'université de Québec.

Plateforme d'échange, de recherche et d'intervention sur la scolarité: persévérance et réussite [PÉRISCOPE]. (2020). Les inégalités sociales et scolaires déjà bien présentes s'accroissent considérablement au temps du Covid-19 : Comment agir dès maintenant? https://www.periscope-

r.quebec/sites/default/files/iniquites_sociales_scolaires_et_actions_avril_2020.pdf

Racine, M. (2020, 27 juillet). Inégalités et iniquités en contexte $\overline{d e}$ COVID-19. Réseau d'information pour la réussite éducative [RIRE]. http://rire.ctreq.qc.ca/2020/07/38936/

Rimm-Kaufman, S. E. \& Pianta, R. C. (2000). An ecological perspective on the transition to kindergarten: A theoretical framework to guide empirical research. Journal of Applied Developmental Psychology, 21(5), 491-511. https://doi.org/10.1016/S01933973(00)00051-4

Rochet, C., Keramidas, O., \& Bout, L. (2008). La crise comme stratégie de changement dans les organisations publiques. Revue Internationale des Sciences Administratives, 74(1), p. 7185. https://www.cairn.info/revue-internationale-des-sciences-administratives-2008-1page-71.htm 
Revue internationale du CRIRES : innover dans la tradition de Vygotsky (2021), vol 5, no 1

Ruel, J. (2020, 2 juin). Introduction. La première transition scolaire en temps de pandémie [Webinaire]. Chantier montréalais transition vers l'école. https://www.reseaureussitemontreal.ca/wpcontent/uploads/2020/05/Pr\%C3\%A9sentation_JRuel_VF_2-juin2020.pdf

Ruel, J., Harvey, N. et Leduc, M.-E. (2020, 2 juin). La première transition scolaire en temps de pandémie [Webinaire]. Chantier montréalais transition vers l'école. https://www.reseaureussitemontreal.ca/wp-content/uploads/2020/05/Webinaire-2-juinChantier-transition.pdf

Ruel, J., Bérubé, A., Moreau, A. \& April, J. (2018). Pratiques déployées lors de la transition vers la maternelle des enfants vivant en milieu défavorisé. La revue internationale de l'éducation familiale, 2(2), 109-129. https://doi.org/10.3917/rief.044.0109

Ruel, J., Moreau, A. C., Bérubé, A., \& April, J. (2015). Les pratiques de transition lors de la rentrée des enfants au préscolaire. Évaluation du « Guide pour soutenir une première transition scolaire de qualité ». Rapport final de recherche. Université du Québec en Outaouais et Pavillon du Parc.

Ruel, J., Moreau, A. C., \& April, J. (2014). Les savoirs en partage entre des acteurs intersectoriels en contexte de transition scolaire. Revue internationale de communication et de socialisation (RICS), 1(1), p. 47-59.

Schlossberg, N. K. \& Goodman, J. (2005). Counseling adults in transition. Linking Schlossberg's Theory With Practice in a Diverse World. Springer.

https://www.springerpub.com/counseling-adults-in-transition-9780826106353.html

Schonfeld, D. J., Demaria, T., \& Kumar, S. M. (2020). Supporting Young Children after Crisis Events. Young Children, 75(3).

https://www.naeyc.org/resources/pubs/yc/jul2020/supporting-young-children-after-crisisevents

Smith, S. \& Maher, M. (2016). The power of play-based learning: A pedagogy of hope for potentially at-risk children. Dans S. Lynch, D. Pike, \& C. Beckett (dir.), Multidisciplinary perspectives on play from early childhood and beyond (p. 181-203). Springer.

https://www.springer.com/gp/book/9789811026416

Ste-Marie, N. (2020). Septembre 2020: Réouverture des écoles par province. Québec, Canada : Centre de recherche et d'intervention sur la réussite scolaire. https://crires.ulaval.ca/sites/default/files/entree_scolaire_par_province_par_nathalie_stemarie_bonne_version.pdf

Stratford, B. (2020). As schools reopen, addressing COVID-19-related trauma and mental health issues will take more than mental health services. Baltimore, MA: Child Trends. https://www.childtrends.org/blog/as-schools-reopen-addressing-covid-19-related-traumaand-mental-health-issues-will-take-more-than-mental-health-services.

Statford, B., Cook, E., Hanneke, R., Katz, E., Seok, D., Steed, H., Fulks, E., Lessans, A., \& Temkin, D. (2020). A Scoping Review of School-Based Efforts to Support Students Who Have Experienced Trauma ». School Mental Health. doi: https://doi.org/10.1007/s12310020-09368-9

Van Laere, K. \& Vandenbroeck, M. (2017). Early learning in preschool: meaningful and inclusive for all? Exploring perspectives of migrant parents and staff. European Early Childhood Education Research Journal, 25(2), 243-257. https://doi.org/10.1080/1350293X.2017.1288017

@2021 Duval, Lehrer, Pirard, Housen 
Wagnon, S. (2020). La continuité pédagogique : méandres et paradoxes en temps de pandémie. Recherches et éducation, HS juillet 2020, 1-19. https://doi.org/10.4000/rechercheseducations. 10451

Watzlawick, P, Weakland, J., \& Fisch, R. (1981). Changements, paradoxes et psychothérapies. Seuil.

White, G. \& Sharp, C. (2007). 'It's different... because you are getting older and growing up'. How children make sense of the transition to year 1. European Early Childhood Education Research Journal, 15(1), 87-102. https://doi.org/10.1080/13502930601161882

Wildenger, L. \& McIntyre, L. (2011). Family concerns and involvement during Kindergarten transition. Journal of Child and Family Studies, 20(4), 387-396. DOI: 10.1007/s10826010-9403-6

Zero to Three. (2020). How COVID-19 is impacting child-care providers. https://www.zerotothree.org/resources/3398-howCOVID-19-is-impacting-child-careproviders. 